Bull. Mater. Sci., Vol. 2, Number 3, August 1980, pp. 181-186. (C) Printed in India.

\title{
Wave-vector dependence of spin-wave line-width in yttrium iron garnets
}

\author{
OM PRAKASH and C M SRIVASTAVA
}

Radar Project Centre, Indian Institute of Technology, Powai, Bombay 400 076, India

MS received 15 May 1980

\begin{abstract}
The wave-vector dependence of the spin-wave line-width in yttrium iron garnets has been studied for samples of grain diameter varying from $1.2 \mu \mathrm{m}$ to $12 \mu \mathrm{m}$. An expression for the spin-wave line-width as a function of wave-vector and grain diameter has been obtained, which agrees satisfactorily with experiment.
\end{abstract}

Keywords. wave-vector; spin wave; yttrinm iron garncts.

\section{Introduction}

The variation of spin-wave line-width $\left(\triangle H_{k}\right)$ and its dependence on the spin-wave vector $(k)$ for different average grain diameter $(d)$ in polycrystalline ferrites and garnets has been discussed by several workers (Vrehen 1966; Patton 1969; Scotter 1972). It is now well established that $\Delta H_{k \rightarrow 0}$ varies inversely with grain diameter $d$ for $2 \leqslant d \leqslant 10 \mu \mathrm{m}$, both in dense as well as porous samples (Patton 1970). This has been qualitatively explained by Vrehen et al (1969) and Patton (1970) on the transit-time independent grain model. In this a spin-wave gets excited inside a grain, propagates and gets annihilated at the grain boundary in a time interval $\tau=d / v_{g}$, where $v_{g}$ is the group velocity of the spin-waves. In this model $v_{g}$ is assumed to be independent of the grain diameters. $\tau$ is experimentally determined from the spin-wave line-width using the relation $\tau=1 / \gamma \triangle H_{k}$ where $\gamma$ is the gyromagnetic ratio and is equal to $1.76 \times 10^{7} \mathrm{Oe}^{-1} \mathrm{sec}^{-1}$.

The study of the dependence of $\triangle H_{z}$ on $k$ shows that $\triangle H_{z}$ varies linearly with $k$ (Patton 1970). The slope of the $\triangle H_{k}$ vs $k$ straight line is a sensitive function of $d$. For large grain size the slope is positive and $\Delta H_{k}$ increases with $k$. As the grain size decreases the slope also decreases and at a critical grain size $\left(d_{0}\right)$, the slope changes its sign and $\Delta H_{k}$ decreases with increase in $k$ (Patton 1970). Patton has attempted to explain this result on the basis of the deviation from $\theta_{k}=\pi / 2$ condition for the instability threshold in the parallel pump experiment. Such an approach leads to a minimum in the $\Delta H_{k}$ vs $k$ curve for small grain diameters which has not been observed for dense samples. 
With a view to understand the possible mechanism which may account for the observed $d$-dependence of the slope we have investigated the $k$-dependence of $\Delta H_{k}$ as a function of average grain size in dense polycrystalline garnets.

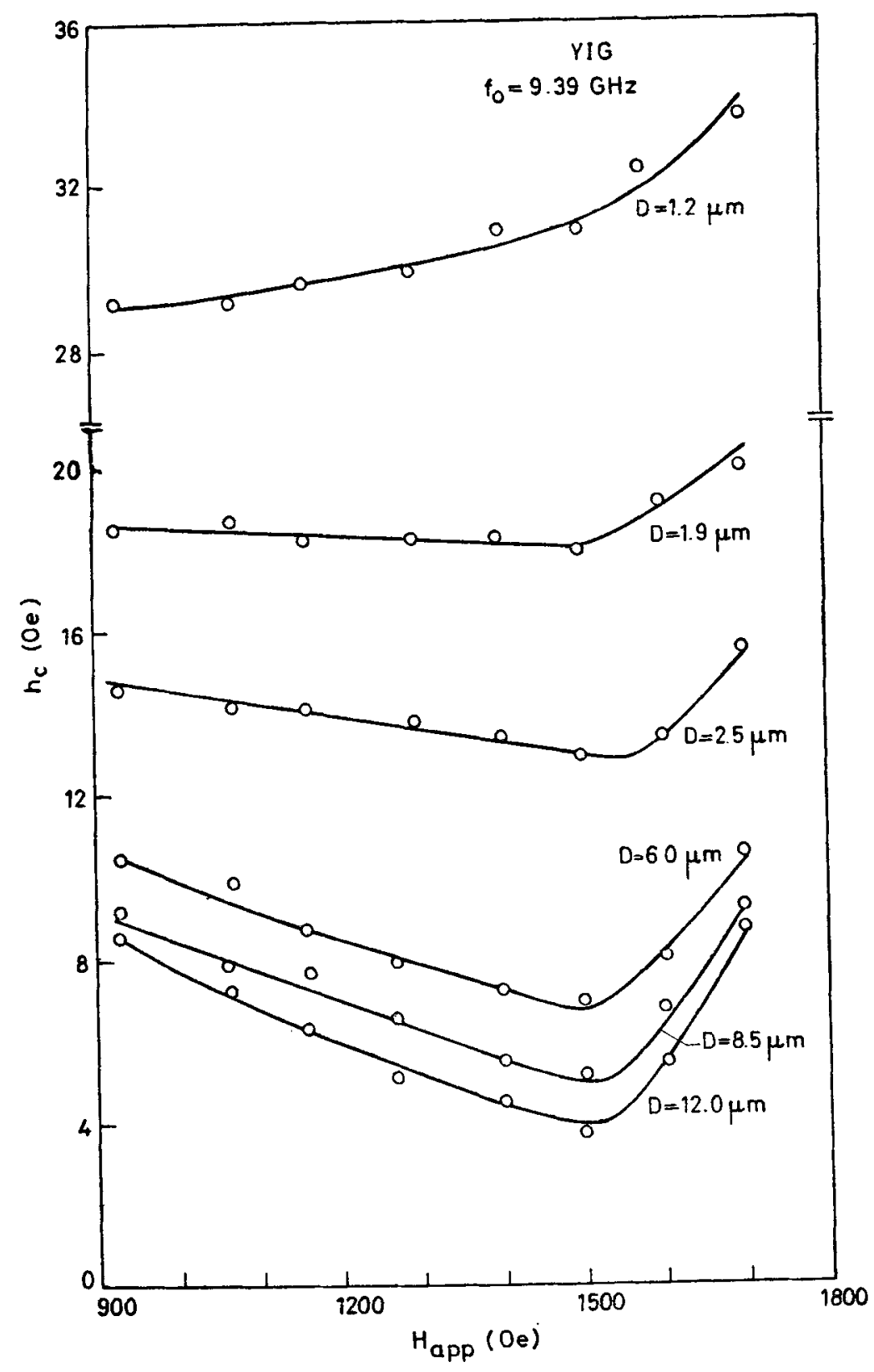

Figure 1. The variation of the critical field $h_{c}$ as a function of the static applied field $H_{\text {app }}$ in YIG at $9.39 \mathrm{GHz}$. The observations were made in the parallel-pump configuration, 


\section{Experimental}

Polycrystalline yttrium iron garnets (YIG) samples with densities greater than $98.5 \%$ of the $\mathrm{x}$-ray density have been prepared using the hot pressing and normal sintering technique. The average grain diameter for these samples ranges from $1 \cdot 2 \mu \mathrm{m}$ to $12 \mu \mathrm{m}$.

A high power microwave bench giving $60 \mathrm{~kW}$ peak power and operating at 9.39 $\mathrm{GHz}$ was used to study the parallel pump instability threshold $\left(h_{e}\right)$ in the polycrystalline samples. The samples were mounted in the centre of a $\mathrm{TE}_{104}$ cavity.

\section{Results}

The instability threshold field $h_{0}$ has been observed as a function of the applied d.c. magnetic field for different grain diameters. The results are given in figure 1 .

The spin-wave line-width $\left(\Delta H_{k}\right)$ is related to the critical field $h_{0}$ through the relation

$$
h_{\boldsymbol{c}}=\omega \Delta H_{k} / \omega_{m} \sin ^{2} \theta_{k}
$$

where $\omega$ is the operating frequency, $\omega_{m}=\gamma 4 \pi M_{s}$ and $\theta_{b}$ is the angle between the k-vector of the spin-wave and the direction of the applied field. The $h_{c}$ vs. $H_{p p}$ curve in figure 1 gives the value of $\Delta H_{k}$ in (1) for $\theta_{k}=\pi / 2$ up to $h_{0}$ min.

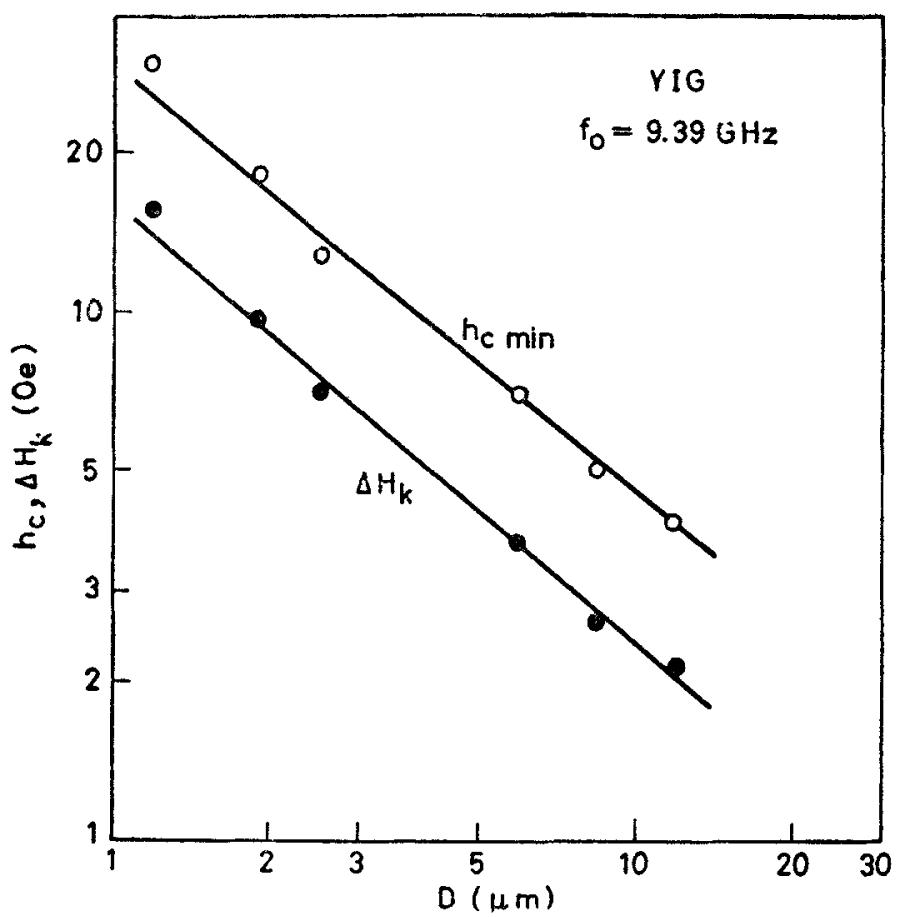

Figure 2. The dependence of the $h_{c \text { min }}$ and $\Delta H_{t}$ on grain diameter in YIG at $9 \cdot 39 \mathrm{GHz}$. The minimum in the critical field occurs for $\theta_{k}=\pi / 2$ magnons. $\triangle H_{k}$ has been obtained from $h_{c}$ nin using equation (1) of the text. 
A $\log$-log plot of $h_{c \min }$ and $\triangle H_{k}$ as a function of grain diameter is given in figure 2. The $k$-dependence of $\Delta H_{k}$ is given in figure 3. In figure 4 is plotted the observed variation of $\Delta H_{k \rightarrow 0}$ with $d^{-1}$.

\section{Discussion}

According to the transit time model

$$
\Delta H_{k}=1 / \gamma \tau=v_{s} / \gamma d
$$

For the parallel pump experiment, $v_{g}=2 \gamma D k$, where $D$ is the exchange stiffness constant. Hence, $\triangle H_{k}=2 D k / d$. The group velocity in fine grain materials for $k \rightarrow 0$ would tend to a constant value which may be given by $2 \gamma D k_{0}$. This

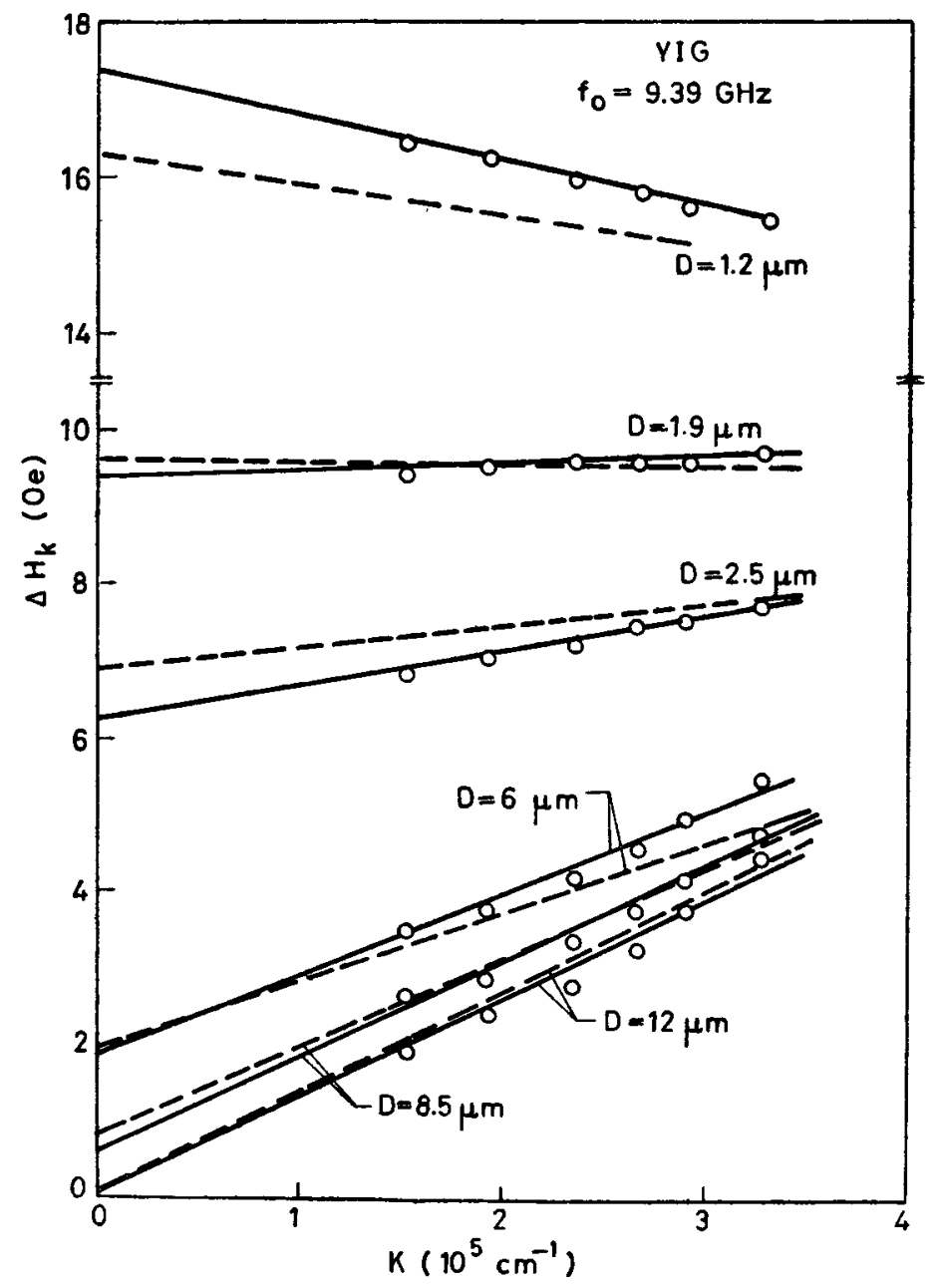

Figure 3. The dependence of $\triangle H_{k}$ on the wave vector $k$ for different values of the grain diameter for YIG. The solid line and the points show the experimental data. The dashed line is the theoretical curve obtained from equation (4) of the text with $D=5 \times 10^{-9} \mathrm{Oe}-\mathrm{cm}^{2}, K_{0}=2.16 \times 10^{5} \mathrm{~cm}^{-1}$ and $C_{0}=-1.7 \mathrm{Oe}$, 


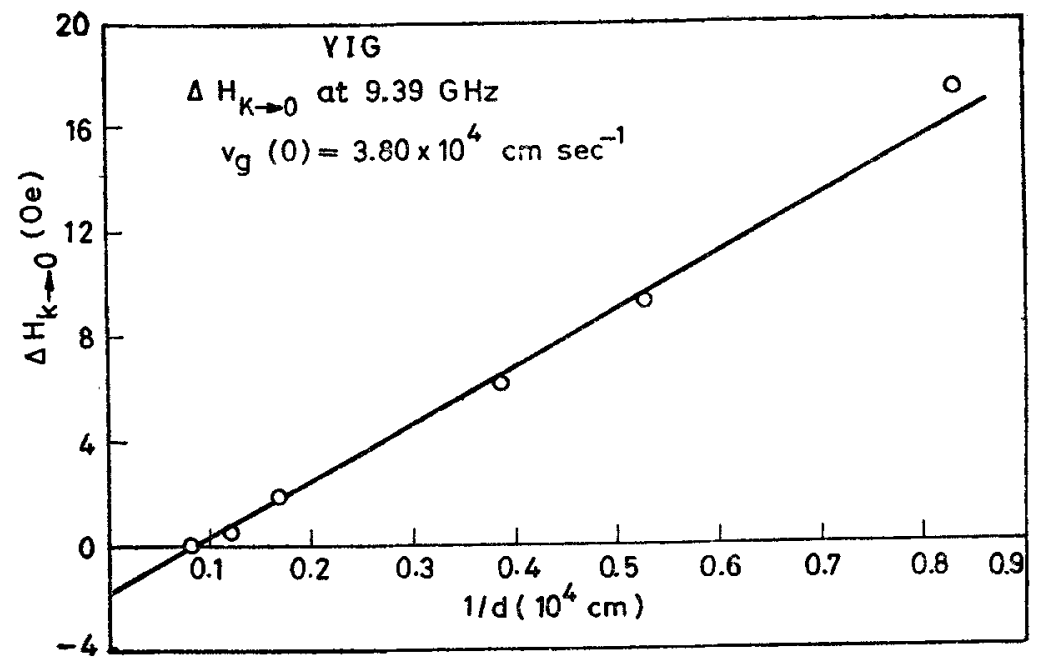

Figure 4. The dependence of the spin-wave line-width for $k \rightarrow 0, \Delta H_{k \rightarrow 0}$ on $d^{-1}$ where $d$ is the grain diameter for YIG.

follows as in the limit $k \rightarrow 0$ the spin wave mode goes over to the magnetostatic mode. Hence we may take.

$$
\triangle H_{k \rightarrow 0}=2 D k_{0} / d+C_{0}
$$

where $C_{0}$ is a constant independent of $d$.

For finite $k$, the experimental observation can be fitted to the following equation,

$$
\Delta H_{k}=\frac{2 D k_{0}}{d}\left[1+\xi(d) \frac{k}{k_{0}}\right]+C_{0},
$$

where

$$
\xi(d)=\frac{d-d_{0}}{d+d_{0}} \quad \frac{d}{3 d_{0}} .
$$

Here $d_{0}$ is a characteristic grain diameter for which the spin-wave line-width becomes independent of $k$.

Using equation (2) and taking the observed variation of $\Delta H_{k \rightarrow 0}$ with $d^{-1}$ given in figure 4 , we obtain

$$
k_{0}=2 \cdot 16 \times 10^{5} \mathrm{~cm}^{-1}
$$

and $\quad C_{0}=-1.7 \mathrm{Oe}$.

Here $D$ has been taken as $5 \times 10^{-9} \mathrm{Oe}-\mathrm{cm}^{2}$, the value appropriate for YIG.

Using the value of $k_{0}$ and $C_{0}$ given in (6) and (7), we have plotted theoretical curves based on (4) in figure 3 and shown them by dashed lines. The agreement with experiment is satisfactory.

\section{Conclusion}

The wave-vector dependence of the spin-wave line-width in YIG has been studied as a function of grain diameter. It has been possible to obtain an expression for 
the spin-wave line-width which is dependent on the grain diameter and the wavevector, and which is in satisfactory agreement with experiment.

\section{References}

Patton C E 1969 Phys. Rev. 179352

Patton C E 1970 J. Appl. Phys. 411637

Scotter D G $1972 J$. Phys. D5 L 93

Vrehen Q H F 1966 J. Appl. Phys. 401849

Vrehen Q H F, Beljers H G and Delau J G M 1969 IEEE Trans. Magn. MAG-5 617 